\title{
Attempts to Improve Antimicrobial Efficiency by Mixed- Lactobacillus Extracts as Crude or Nano-formulated Against Pathogenic and Food Spoilage Bacteria, Molds and Yeasts
}

\author{
Mohamed F. El-Ssayad ${ }^{1, *(\mathbb{D})}$, Gamal A. Ibrahim ${ }^{2 \mathbb{D}}$, Osama M. Sharaf ${ }^{3(\mathbb{D})}$ \\ 1 Dairy Science Dept., (Dairy Microbiol. Lab.), National Research Centre, 33 El-Bohouth St. (Former El-Tahrir St.) Dokki, Giza, \\ Egypt; sayad.nrc2012@gmail.com (M.F.E.); gamalwahab2015@gmail.com (G.A.I.); sharafosama@yahoo.com (O.M.S.); \\ * Correspondence: sayad.nrc2012@gmail.com (M.F.E);
}

Scopus Author ID: 57073976000

Received: 5.07.2021; Revised: 29.08.2021; Accepted: 3.09.2021; Published: 18.10.2021

Abstract: Over the world, there are millions of disease cases, in addition to thousands of deaths due to food-based outbreaks every year. In order to diminish the risk of foodborne infection and intoxications and control microbial food spoilage, there is a great need to improve modes of food preservation. As wellknown, nature-dependent preservation enables consumers to keep their safety while eating highly qualified food. This work aims to enhance the safety and quality of common popular food products by introducing new preservative applications suggestions. The idea is to use different lactobacilli-based fermentations; in single or mixed-status and study the obtained extracts to evaluate their influence to manage microbial contamination of food. A set of five Lactobacillus strains was utilized by this study, methanol extract of cold acetone precipitate, Diethyl ether extract, and both were tested as crude or Nano-formulated against common microbial pathogens and food spoilers. Methanol extract of all lactobacillus strains shows antibacterial-specific activity with inhibition zone ranging from $7 \mathrm{~mm}$ in the case of Lactobacillus rhamnosus against Salmonella enterica, to $24 \mathrm{~mm}$ in the case of Lactobacillus helveticus against Escherichia coli. Diethyl ether extracts based on Lactobacillus helveticus and Lactobacillus plantarum show a considerable inhibition to all utilized microbial concerns, including Saccharomyces cerviceae. Upon estimating three forms of mixed-Lactobacillus that are based on Lactobacillus helveticus (h), Lactobacillus plantarum (p), and Lactobacillus rhamnosus $\mathrm{GG}(\mathrm{G})$, the ph-dependant combination showed uprising inhibition that reached maximally 58\% over that of single Lactobacilli. Nano formulation of mixed-based total extracts maximally upraised the inhibition to $39 \%, 32 \%$, and $100 \%$ against gram-positive, gramnegative, and the fungus; Penicillium chrysogenum, respectively.

Keywords: antimicrobial activity; nanoparticles; pathogenic and spoilage microorganisms; single and mixed-Lactobacillus fermentation; food biopreservation.

(C) 2021 by the authors. This article is an open-access article distributed under the terms and conditions of the Creative Commons Attribution (CC BY) license (https://creativecommons.org/licenses/by/4.0/).

\section{Introduction}

Because there is no zero-risk system, foodborne diseases and intoxications are in progress. The Council for Agricultural Science and Technology reported that foodborne pathogens cause an estimated 6.5-33 million cases of human illness and up to 9000 deaths annually, the main foods implicated being meat, poultry, eggs, seafood, and dairy products [1, 2]. Awareness in biopreservation has increased worldwide, supported by research results that protective cultures or https://biointerfaceresearch.com/ 
their inhibitory metabolites may have bio-preservative potential to render pathogenic populations in foods [3,4]. These pathogens that account for most of these cases include Salmonella, Campylobacter jejuni, Escherichia coli, Listeria monocytogenes, Staphylococcus aureus, and Clostridium botulinum [5, 6]. Fungi, the public food spoilers, are common concerns in dairy products, where a favorable niche for their growth exists. There is great attention to control fungal spoilage through searching for efficient solutions [7-9]. Many conventional methods called traditional hurdle technologies are applied in combinations to achieve real preservation. Microbial control and prevention include good manufacturing practices, proper hygiene, inactivation treatments, temperature control, and modified atmosphere packaging [7, 10, 11].

Lactobacilli are known to produce antibacterial compounds, including bacteriocins and bacteriocin-like peptides [12-15]. Most of the bacteriocins produced by Lactobacillus species are small, thermally stable proteins, known as type II bacteriocins [16] which efficiently affect some antibiotic-resistant microbes [17]. These compounds can induce rupture of the cell membrane, causing leakage of cell contents and playing a role in sterilization [18].

Lactobacilli produce various compounds such as organic acids, diacetyl, hydrogen peroxide, and bacteriocin during lactic fermentations. All of these can antagonize the growth of some spoilage and pathogenic bacteria in foods [19]. In particular, there has been a renewed interest in the antimicrobial activity of lactobacilli, which has been important for centuries in the preservation of food. Probiotic Lb. rhamnosus strain shows a broad spectrum of activity against gastrointestinal tract pathogens and food spoilage organisms [20]. Lactobacilli drive the inhibitory process through the production of organic acids (primarily lactic and acetic acid), hydrogen peroxide, bacteriocins, and other substances [21-23], showing strong antagonistic activity against spoilage and pathogenic bacteria and fungi [24].

Nanoparticles can be applied to control the delivery of antimicrobial compounds in food and nonfood microbial safety applications [25]. Antimicrobial compounds can be attached to cores of nanoparticles and delivered into bacterial cells. The antibacterial mechanisms of NPs are not completely understood, but oxidative stress induction, metal ion release, and non-oxidative theory are the most accepted [26-28].

The aim of the present investigation was to assess the perfect combination of nano or crude lactobacilli cell-free supernatant (Lactobacillus helveticus, Lb. plantarum, Lb. reuteri, Lb. rhamnosus, Lb. rhamnosus GG) that gives rise to an antimicrobial mixture useful to control pathogenic and spoilage bacteria, molds, and yeasts.

\section{Materials and Methods}

\subsection{Bio-protective strains.}

Lactobacillus plantarum DSA 20174 is provided by Cairo MIRCEN, Faculty of Agriculture, Ain Shams University, Egypt; Lactobacillus rhamnosus NRRL B-442 and Lactobacillus reuteri NRRL B-14171 are obtained from the Northern Regional Research Laboratory, Illinois; USA. Lactobacillus helveticus CNRZ 32 Collection of dairy Microbiological Laboratory (supplemented from Centre National de Recherché Zootechnique, Jouy-en-Josas, France). Lactobacillus rhamnosus GG was supplemented by Afify et al., [29] from the collection of the Food sciences \& Nutrition dept., NRC. 


\subsection{Pathogenic and food spoilage microorganisms.}

Escherichia coli (E. coli) strain E11 (accession number KY780346.1), Salmonella enterica (S. enterica) strain SA19992307 (accession number CP030207.1), Pseudomonas aeruginosa (Ps. aeruginosa) strain Kasamber5 (accession number KY549641.1), Bacillus cereus (B. cereus) strain 151007-R3-K09-40-27F (accession number KY820914.1) were isolated and identified by AlGamal et al., [30]. Listeria monocytogenes (L. monocytogenes) strain was supplemented from the collection of Dairy Microbiological Lab., NRC, Egypt; Staphylococcus aureus (S. aureus) is a clinical isolate, while Aspergillus flavus (Asp. flavus)3357 and Saccharomyces cerviceae (Sac. Cerviceae) Y-2223 were provided by the Northern Regional Research Laboratory Illinois, USA (NRRL).

\subsection{Materials.}

Skimmed milk was purchased from animal production Research Institute, Agricultural Research Centre, Egypt; MRS broth is purchased from SRL, India; Nutrient agar is provided from Panreac Quimica, Spain; Malt extract agar is imported from Biolife, Italy; Chitosan (M.W: 100.000 - 300.000) is purchased from ACROS ORGANICS, UK; Acetone is purchased from ADWIC, Egypt; Methanol (HPLC grade) is supplemented by Fisher Scientific, UK.

\subsection{Precipitation of active peptides by cold acetone.}

The antimicrobial peptides are concentrated from $150 \mathrm{ml}$ of Cell-free supernatant via cold acetone extraction. Four times the sample volume of cold $\left(-20^{\circ} \mathrm{C}\right)$ acetone is added to the cooled sterile supernatant sample $\left(4^{\circ} \mathrm{C}\right)$, mixed for $15 \mathrm{~min}$, and incubated $60 \mathrm{~min}$ at $4^{\circ} \mathrm{C}$. The pellet is collected by further centrifugation and extracted in $50 \mathrm{ml}$ methanol with stirring for 2 hours [31]. Evaporation of methanol is carried out using a rotary evaporator until peptide dry film is obtained.

\subsection{Characterization of Methanol extract}

\subsubsection{Fourier-Transform Infrared Spectroscopy (FT-IR) analysis.}

The absorbance FT-IR spectra of the samples are documented using an FT-IR PerkinElmer spectrometer. The spectra are collected within a scanning range of $400-4000 \mathrm{~cm}^{-1}$, Central Lab. for Services, NRC, Dokki, Giza, Egypt.

\subsubsection{GC/MS analysis of diethyl ether extract.}

The analysis was performed in a chromatographic laboratory, central laboratories network, National Research Centre, Dokki, Egypt, using a GC-MS system (7890A-5975C, Agilent Technologies Inc., Santa Rosa, CA, USA) equipped with an HP-5 MS capillary column (30 $\mathrm{m} \times$ $0.25 \mathrm{~mm}, 0.25 \mathrm{~mm}$, Agilent Technologies Inc., Santa Rosa, CA, USA). Exactly, $1 \mu \mathrm{L}$ of the sample was injected using Helium (99.999\%) - as the carrier- at $1 \mathrm{ml} / \mathrm{min}$. The injection port temperature was $280{ }^{\circ} \mathrm{C}$, and the column temperature cycle was $40{ }^{\circ} \mathrm{C}$ for $5 \mathrm{~min}$, followed by an increase to $150{ }^{\circ} \mathrm{C}$ at a rate of $5^{\circ} \mathrm{C} / \mathrm{min}$, and an increase to $210^{\circ} \mathrm{C}$ at the rate of $10^{\circ} \mathrm{C} / \mathrm{min}$. The MS conditions Capillary column and 5975B Inert XL MS system under electron ionization at $70 \mathrm{eV}$ 
and Quadrupole mass analyzer. The MS source and Quadrupole were held at $230{ }^{\circ} \mathrm{C}$ and $150{ }^{\circ} \mathrm{C}$, respectively, using Helium as carrier gas as follows.

\subsubsection{Preparation and characterization of loaded-nanoparticles.}

Chitosan nanoparticles (Ch.-NPs) are prepared by dissolving 2 Grams of chitosan in 1\% acetic acid solution. After complete dissolution, chitosan solution is added drop-wisely to the vigorously stirred Sodium Tri-polyphosphate (TPP) solution $(0.03 \%)$. The resulted suspension is then subjected to sonication (sonication power, $750 \mathrm{Watts}$, frequency, $20 \mathrm{kHz}$ and amplitude $50 \%$, for 30 minutes at $25^{\circ} \mathrm{C}$. Nanoparticles are stabilized by the addition of $0.4 \%$ Cetyltrimethylammonium bromide (CTAB) as a cationic surfactant. Nanoemulsions were prepared by mixing one part of extract into two parts of both $\mathrm{H}_{2} \mathrm{O}$ and Tween 80 .

The particle size distribution and zeta potential were estimated in central laboratories network, National Research Centre, Dokki, Egypt using Particle Sizing Systems, Inc. Santa Barbara, Calif., USA.

\subsubsection{Antimicrobial assay.}

Firstly, the antimicrobial activity of the crude peptide extract that had been dissolved in 0.2 $\mathrm{ml}$ of DMSO and extract-loaded chitosan NPs. is evaluated through disc diffusion assay as recommended in the British Society for Antimicrobial Chemotherapy guidelines [32]. Briefly, a typical colony was picked and introduced in a $5 \mathrm{ml}$ of tryptone soy broth from the overnight incubated culture. The broth culture was incubated at $35^{\circ} \mathrm{C}$ until visible turbidity reached 0.5 "McFarland" standard solution. Then, nutrient agar plates $(25 \mathrm{ml}$ agar / 9cm plate or equivalent) were inoculated with sterile cotton swabs in three directions to give a semi-confluent growth after overnight incubation finally. Within 15 minutes, discs with tested substances were applied on the dried surface of the inoculated agar plates. After incubation at $35^{\circ} \mathrm{C}$ for $20 \mathrm{~h}$, inhibition zone diameters $(\mathrm{mm})$ were recorded.

\subsubsection{Statistical analysis.}

Statistical significance was determined using Statistica Version 9 (State Soft, Tulsa, Okla., USA). The means were determined by analysis of variance test (ANOVA, two-way analysis) $(\mathrm{p}<0.05)$ [33].

\section{Results and Discussion}

\subsection{Antimicrobial activity.}

3.1.1. Methanol extract of cold acetone precipitates.

Lactobacillus fermentates were treated with cold acetone and underwent centrifugation. The resulting precipitates were then extracted with methanol. The disc diffusion method estimated the inhibitory activity of lactobacillus extracts against the common food microbial concerns, and the results are presented in Table 1 . It is clear that the tested extracts have a strictly antibacterial effect with no activity against molds nor yeasts. Among all LAB cultures, the tested peptides of 
Lb. helveticus and Lb. rhamnosus GG show higher antimicrobial activity against all bacterial strains.

Table 1. Inhibition zone ( $\mathrm{mm})$ of single Lactobacilli methanol extract.

\begin{tabular}{|c|c|c|c|c|c|c|c|}
\hline & $\begin{array}{c}\text { Lb. } \\
\text { helveticus }\end{array}$ & $\begin{array}{c}\text { Lb. } \\
\text { rhamnosus } \\
\text { GG }\end{array}$ & $\begin{array}{c}\text { Lb. } \\
\text { rhamnosus }\end{array}$ & Lb. reuteri & $\begin{array}{c}\text { Lb. } \\
\text { plantarum }\end{array}$ & $\mathrm{C}+$ & C- \\
\hline B. cereus & $15.0 \pm 0.96^{\mathbf{A}}$ & $14.0 \pm 0.96^{\mathrm{A}}$ & $11.0 \pm 0.00^{\mathbf{D}}$ & $11.0 \pm 0.00^{\mathbf{D}}$ & $11.0 \pm 0.28^{\mathbf{D}}$ & $13.0 \pm 0.00^{\mathrm{M}}$ & $0.0 \pm 0.00^{\mathrm{H}}$ \\
\hline S. aureus & $15.0 \pm 0.28^{\mathrm{A}}$ & $10.0 \pm 0.00^{\mathrm{C}}$ & $10.0 \pm 0.00^{\mathrm{C}}$ & $0.0 \pm 0.00^{\mathrm{H}}$ & $8.0 \pm 0.00^{F}$ & $17.0 \pm 0.00^{\mathbf{B}}$ & $0.0 \pm 0.00^{\mathbf{H}}$ \\
\hline L. monocytogenes & $18.0 \pm 0.28^{\mathbf{B}}$ & $12.0 \pm 0.48^{\mathbf{D}}$ & $11.0 \pm 0.48^{\mathbf{D}}$ & $10.0 \pm 0.00^{\mathrm{C}}$ & $7.0 \pm 0.00^{\mathbf{G}}$ & $9.0 \pm 0.00^{\mathbf{F}}$ & $0.0 \pm 0.00^{\mathbf{H}}$ \\
\hline E. coli & $24.0 \pm 0.96^{\mathrm{K}}$ & $13.0 \pm 0.00^{\mathrm{M}}$ & $9.0 \pm 0.00^{\mathrm{F}}$ & $14.0 \pm 0.48^{\mathbf{A}}$ & $18.0 \pm 0.96^{\mathbf{B}}$ & $20.0 \pm 0.00^{\mathbf{E}}$ & $0.0 \pm 0.00^{\mathrm{H}}$ \\
\hline S. enterica & $12.0 \pm 0.00^{\mathbf{D}}$ & $16.0 \pm 0.00^{A}$ & $7.0 \pm 0.00^{\mathbf{G}}$ & $18.0 \pm 0.48^{\mathbf{B}}$ & $12.0 \pm 0.96^{\mathbf{D}}$ & $12.0 \pm 0.00^{\mathbf{D}}$ & $0.0 \pm 0.00^{\mathrm{H}}$ \\
\hline Ps. aeruginosa & $11.3 \pm 0.28^{\mathbf{D}}$ & $0.0 \pm 0.00^{\mathrm{H}}$ & $0.0 \pm 0.00^{\mathrm{H}}$ & $0.0 \pm 0.00^{\mathrm{H}}$ & $0.0 \pm 0.00^{\mathrm{H}}$ & $0.0 \pm 0.00^{\mathrm{H}}$ & $0.0 \pm 0.00^{\mathrm{H}}$ \\
\hline Asp. flavus & $0.0 \pm 0.00^{\mathbf{H}}$ & $0.0 \pm 0.00^{\mathrm{H}}$ & $0.0 \pm 0.00^{\mathrm{H}}$ & $0.0 \pm 0.00^{\mathrm{H}}$ & $0.0 \pm 0.00^{\mathrm{H}}$ & $9.0 \pm 0.00^{\mathrm{F}}$ & $0.0 \pm 0.00^{\mathrm{H}}$ \\
\hline Pen. chrysogenum & $0.0 \pm 0.00^{\mathrm{H}}$ & $0.0 \pm 0.00^{\mathrm{H}}$ & $0.0 \pm 0.00^{\mathrm{H}}$ & $0.0 \pm 0.00^{\mathrm{H}}$ & $0.0 \pm 0.00^{\mathrm{H}}$ & $12.0 \pm 0.00^{\mathbf{D}}$ & $0.0 \pm 0.00^{\mathrm{H}}$ \\
\hline Sac. cerviceae & $0.0 \pm 0.00^{\mathrm{H}}$ & $0.0 \pm 0.00^{\mathrm{H}}$ & $0.0 \pm 0.00^{\mathrm{H}}$ & $0.0 \pm 0.00^{\mathrm{H}}$ & $0.0 \pm 0.00^{\mathrm{H}}$ & $12.0 \pm 0.00^{\mathbf{D}}$ & $0.0 \pm 0.00^{\mathrm{H}}$ \\
\hline
\end{tabular}

The tested extracts of all studied Lactobacilli caused a considerable inhibition to all bacterial pathogens. Inhibition zone diameter ranged from $7 \mathrm{~mm}$ to $24 \mathrm{~mm}$, depending on both pathogenic and Lactobacillus strains. At $\mathrm{P}<0.05$, among all Lactobacilli, the extract of $L b$. helveticus only affected the growth of Ps. aeruginosa $(11.3 \mathrm{~mm})$.

\subsubsection{Diethyl ether extracts (DEE) of single Lactobacilli fermentates.}

Disc diffusion assay of the Diethyl ether extracts was performed to define their inhibitory activity against different pathogens (Figure 1).

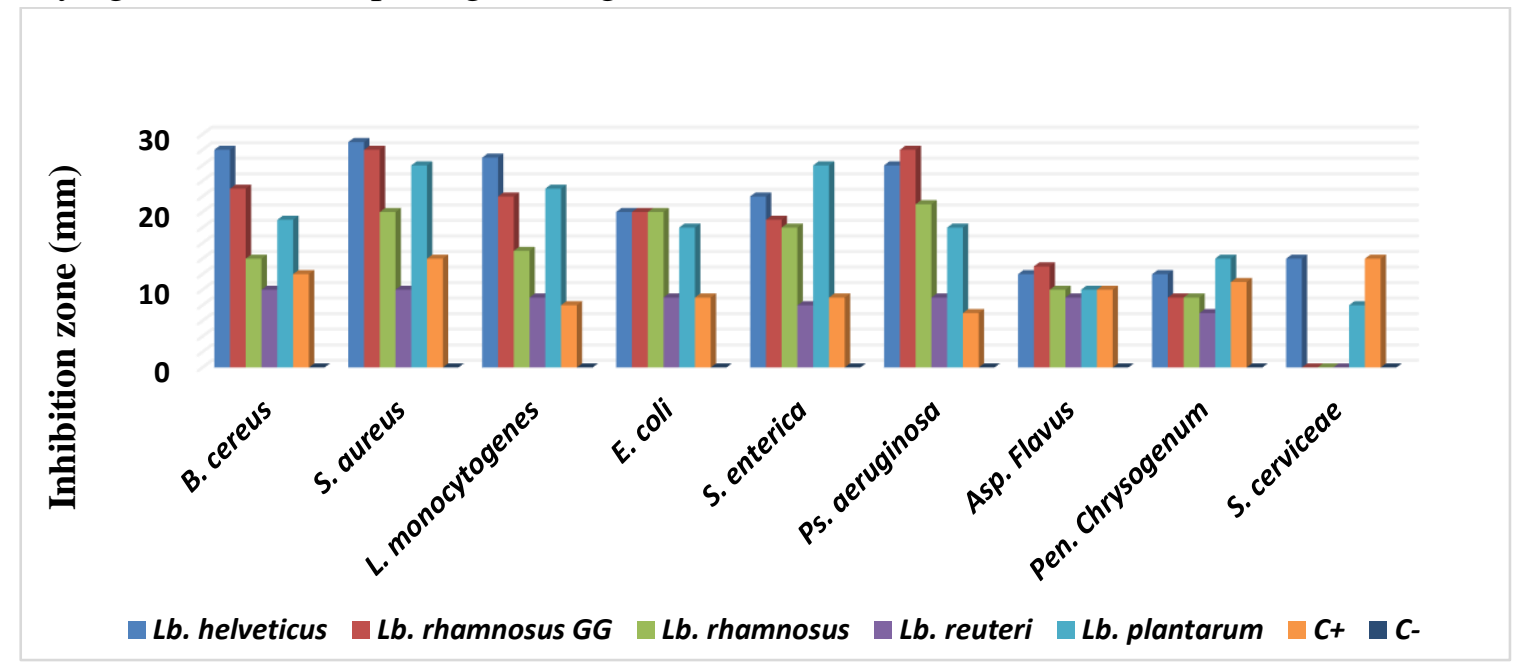

Figure 1. Inhibitory effect of Diethyl ether extract of single Lactobacilli fermentate.

As observed, the growth of all pathogenic and food spoilage strains was inhibited by DEE of studied Lactobacilli. The diameter of inhibition zones came in the range of $7 \mathrm{~mm}$ to $29 \mathrm{~mm}$. Only Saccharomyces cerviceae showed no response (not inhibited) to all Lactobacilli except Lactobacillus helveticus and Lactobacillus plantarum, which induced inhibition diameter of $9 \mathrm{~mm}$ and $18 \mathrm{~mm}$ respectively.

\subsubsection{Total extract of single Lactobacilli fermentates.}


Data in Table 2 describe the degree of antimicrobial effect due to the application of lactobacilli total extract (Methanol extract + DEE).

Table 2. Inhibition zone (mm) of total extracts of single Lactobacilli

\begin{tabular}{|c|c|c|c|c|c|c|c|}
\hline & $\begin{array}{c}\text { Lb. } \\
\text { helveticus }\end{array}$ & $\begin{array}{c}\text { Lb. } \\
\text { rhamnosus } \\
\text { GG }\end{array}$ & $\begin{array}{c}\text { Lb. } \\
\text { rhamnosus }\end{array}$ & Lb. reuteri & $\begin{array}{c}L b . \\
\text { plantarum }\end{array}$ & $\mathrm{C}+$ & $\mathrm{C}-$ \\
\hline B. cereus & $39 \pm 1.44^{\mathbf{E}}$ & $33 \pm 0.96^{\mathbf{G}}$ & $35 \pm 0.48^{\mathrm{K}}$ & $35 \pm 1.44^{\mathrm{K}}$ & $30 \pm 0.48^{\mathrm{H}}$ & $12 \pm 0.00^{\mathrm{M}}$ & $0.0 \pm 0.00^{\mathbf{D}}$ \\
\hline S. aureus & $43 \pm 0.00^{\mathbf{F}}$ & $40 \pm 0.00^{\mathbf{E}}$ & $40 \pm 0.00^{\mathbf{E}}$ & $28 \pm 0.50^{\mathbf{B}}$ & $30 \pm 0.00^{\mathrm{H}}$ & $13 \pm 0.00^{\mathbf{M}}$ & $0.0 \pm 0.00^{\mathbf{D}}$ \\
\hline L. monocytogenes & $29 \pm 0.70^{\mathrm{H}}$ & $32 \pm 0.00^{\mathrm{G}}$ & $32 \pm 0.00^{\mathbf{G}}$ & $23 \pm 0.00^{\mathrm{C}}$ & $30 \pm 0.00^{\mathrm{H}}$ & $13 \pm 0.00^{\mathrm{M}}$ & $0.0 \pm 0.00^{\mathbf{D}}$ \\
\hline E. coli & $33 \pm 0.00^{\mathbf{G}}$ & $30 \pm 0.00^{\mathrm{H}}$ & $23 \pm 0.00^{\mathbf{G}}$ & $18 \pm 0.00^{\mathbf{A}}$ & $27 \pm 0.50^{\mathbf{B}}$ & $0.0 \pm 0.00^{\mathbf{D}}$ & $0.0 \pm 0.00^{\mathbf{D}}$ \\
\hline S. enterica & $33 \pm 0.00^{\mathbf{G}}$ & $36 \pm 0.00^{\mathrm{K}}$ & $28 \pm 0.00^{\mathrm{H}}$ & $28 \pm 0.00^{\mathrm{H}}$ & $30 \pm 0.00^{\mathrm{H}}$ & $18 \pm 0.00^{\mathrm{A}}$ & $0.0 \pm 0.00^{\mathbf{D}}$ \\
\hline Ps. aeruginosa & $35 \pm 0.69^{\mathrm{K}}$ & $32 \pm 0.00^{\mathbf{G}}$ & $24 \pm 1.00^{\mathrm{C}}$ & $20 \pm 0.00^{Z}$ & $32 \pm 0.00^{\mathbf{G}}$ & $16 \pm 0.00^{\mathbf{R}}$ & $0.0 \pm 0.00^{\mathrm{D}}$ \\
\hline Asp. flavus & $12 \pm 0.96^{M}$ & $14 \pm 1.44^{\mathrm{M}}$ & $9 \pm 0.48^{\mathbf{S}}$ & $9 \pm 0.48^{\mathbf{S}}$ & $11 \pm 0.96^{\mathrm{M}}$ & $12 \pm 0.28^{\mathrm{M}}$ & $0.0 \pm 0.00^{\mathbf{D}}$ \\
\hline Pen. chrysogenum & $15 \pm 0.96^{\mathbf{R}}$ & $15 \pm 1.44^{\mathbf{R}}$ & $8 \pm 0.48^{\mathrm{S}}$ & $16 \pm 1.44^{\mathbf{R}}$ & $13 \pm 0.96^{\mathbf{M}}$ & $10 \pm 0.48^{\mathrm{S}}$ & $0.0 \pm 0.00^{\mathrm{D}}$ \\
\hline Sac. cerviceae & $15 \pm 0.00^{\mathbf{R}}$ & $0.0 \pm 0.00^{\mathrm{D}}$ & $0.0 \pm 0.00^{\mathrm{D}}$ & $0.0 \pm 0.00^{\mathrm{D}}$ & $10 \pm 0.00^{\mathrm{S}}$ & $15 \pm 0.00^{\mathbf{R}}$ & $0.0 \pm 0.00^{\mathrm{D}}$ \\
\hline
\end{tabular}

As observed, there is a general extension and enhancement of inhibition ranging from 9 $\mathrm{mm}$ (in the case of Lactobacillus rhamnosus and Lactobacillus reuteri) to $43 \mathrm{~mm}$ (in case of Lactobacillus helveticus) against all pathogenic and spoilage strains. In the case of Saccharomyces cerviceae, just Lactobacillus helveticus and Lactobacillus plantarum still inhibiting the yeast growth by $15 \mathrm{~mm}$ and $10 \mathrm{~mm}$ respectively.

\subsubsection{Different extracts of Mixed-Lactobacilli fermentates.}

As their antimicrobial behavior is promising, all of Lactobacillus helveticus, Lactobacillus plantarum and Lactobacillus rhamnosus GG have undergone a set of combinations to inspect the effect of mixed-Lactobacillus fermentation on inhibitory potential against the group of pathogenic and food spoiler organisms. Three combinations, including Lactobacillus helveticus : Lactobacillus plantarum (ph 1:1); Lactobacillus helveticus : Lactobacillus plantarum : Lactobacillus rhamnosus GG (phG 1:1:1); and Lactobacillus helveticus : Lactobacillus rhamnosus GG (hG 1:1) were generated. The resulted data of different extracts from mixed-Lactobacillus fermentation are presented in Table 3 . Regarding the total extract, there is a general incrementexcept small declining against some pathogens- in the inhibitory effect of all extracts belonging to the three combinations. Significantly, there is no difference in activity against B. cereus among the three Lactobacilli combinations.

At $\mathrm{P}<0.05$, the ph-based combination shows a significant enhancement in the activity, ranging from $16.7 \%$ and $13.3 \%$ with molds; Asp. flavus and Pen. chrysogenum to $\sim 58 \%$ in the case of Listeria monocytogenes. The observed decline in the activity is seen with S. aureus, Ps. aeruginosa, and Sac. cerviceae as $21 \%, 5.7 \%$, and $46.7 \%$, respectively. Within hG-based combination, inhibitory activity is still improving to reach $\sim 33.3 \%$ with Pen. chrysogenum, $28.6 \%$ with $P$ s. aeruginosa, and $33.33 \%$ with E. coli, but activity is reduced by $24.6 \%$ in the case of $S$. aureus. Upon evaluation of phG-based mixture, the resulted activity is severely decreased, especially with S. aureus (30.2\%) and Ps. aeruginosa (20\%). 
Table 3. Inhibitory potential of different extracts from mixed-Lactobacillus fermentation.

\begin{tabular}{|c|c|c|c|c|c|c|c|c|c|c|c|}
\hline & & & & \multirow{2}{*}{\multicolumn{3}{|c|}{ phG Combination }} & \multirow{2}{*}{\multicolumn{3}{|c|}{ hG Combination }} & \multirow[b]{3}{*}{$\mathrm{C}+$} & \multirow[b]{3}{*}{ C- } \\
\hline & \multicolumn{3}{|c|}{ ph Combination } & & & & & & & & \\
\hline & DEE extract & $\begin{array}{c}\text { Methanol } \\
\text { extract }\end{array}$ & $\begin{array}{c}\text { Total } \\
\text { extract }\end{array}$ & $\begin{array}{c}\text { DEE } \\
\text { extract }\end{array}$ & $\begin{array}{c}\text { Methanol } \\
\text { extract }\end{array}$ & $\begin{array}{c}\text { Total } \\
\text { extract }\end{array}$ & DEE extract & $\begin{array}{c}\text { Methanol } \\
\text { extract }\end{array}$ & $\begin{array}{c}\text { Total } \\
\text { extract }\end{array}$ & & \\
\hline B. cereus & $36 \pm 1.44^{\mathbf{A}}$ & $26 \pm 0.00^{\mathrm{C}}$ & $43 \pm 0.00^{\mathrm{T}}$ & $34 \pm 0.00^{\mathbf{A}}$ & $22 \pm 0.00^{\mathrm{C}}$ & $42 \pm 0.00^{\mathrm{T}}$ & $39.3 \pm 1.47^{\mathbf{R}}$ & $23 \pm 0.00^{\mathrm{C}}$ & $43.7 \pm 0.99^{\mathrm{T}}$ & $14 \pm 0.00^{\mathrm{E}}$ & $0.0 \pm 0.00^{\mathrm{H}}$ \\
\hline Staph. aureus & $32.7 \pm 1.54^{\mathrm{A}}$ & $18 \pm 0.00^{\mathbf{D}}$ & $34 \pm 0.00^{\mathbf{A}}$ & $30 \pm 0.00^{\mathbf{B}}$ & $16 \pm 0.00^{\mathbf{E}}$ & $30 \pm 0.00^{\mathbf{B}}$ & $33 \pm 0.00^{\mathrm{A}}$ & $18 \pm 0.00^{\mathbf{D}}$ & $32.4 \pm 1.54^{\mathrm{A}}$ & $15 \pm 0.00^{\mathrm{E}}$ & $0.0 \pm 0.00^{\mathrm{H}}$ \\
\hline L. monocytogenes & $30 \pm 0.00^{\mathbf{B}}$ & $27 \pm 1.00^{\mathrm{C}}$ & $46 \pm 1.73^{\mathbf{P}}$ & $32 \pm 0.00^{\mathbf{A}}$ & $24 \pm 0.00^{\mathrm{C}}$ & $42 \pm 0.00^{\mathrm{T}}$ & $35 \pm 0.00^{\mathbf{A}}$ & $29 \pm 0.70^{\mathrm{C}}$ & $47 \pm 1.73^{\mathbf{P}}$ & $10 \pm 0.00^{\mathbf{G}}$ & $0.0 \pm 0.00^{\mathrm{H}}$ \\
\hline E. coli & $35 \pm 0.00^{\mathrm{A}}$ & $16 \pm 0.00^{\mathbf{E}}$ & $38 \pm 0.00^{\mathbf{R}}$ & $27 \pm 0.00^{\mathbf{C}}$ & $20 \pm 0.00^{\mathbf{D}}$ & $37.7 \pm 1.47^{\mathrm{A}}$ & $31 \pm 0.00^{\mathbf{B}}$ & $13 \pm 0.00^{\mathbf{G}}$ & $44 \pm 0.83^{\mathrm{T}}$ & $8 \pm 0.00^{\mathrm{K}}$ & $0.0 \pm 0.00^{\mathrm{H}}$ \\
\hline Sal. enterica & $37 \pm 0.00^{\mathbf{A}}$ & $22 \pm 1.11^{\mathbf{D}}$ & $39.5 \pm 0.00^{\mathbf{R}}$ & $33 \pm 0.00^{\mathrm{A}}$ & $22 \pm 0.00^{\mathrm{C}}$ & $34.8 \pm 0.48^{\mathbf{A}}$ & $36 \pm 0.00^{\mathbf{A}}$ & $21 \pm 0.00^{\mathrm{D}}$ & $39 \pm 0.99^{\mathbf{R}}$ & $10 \pm 0.00^{\mathbf{G}}$ & $0.0 \pm 0.00^{\mathrm{H}}$ \\
\hline Ps. aeruginosa & $31.6 \pm 1.11^{\mathrm{B}}$ & $16 \pm 0.00^{\mathbf{E}}$ & $33 \pm 0.00^{\mathbf{A}}$ & $29 \pm 0.99^{\mathrm{C}}$ & $18 \pm 0.00^{\mathbf{D}}$ & $28 \pm 0.28^{\mathrm{C}}$ & $35 \pm 0.00^{\mathbf{A}}$ & $18 \pm 0.00^{\mathbf{D}}$ & $45 \pm 0.00^{\mathbf{P}}$ & $0 \pm 0.00^{\mathbf{H}}$ & $0.0 \pm 0.00^{\mathrm{H}}$ \\
\hline Asp. flavus & $23 \pm 1.73^{\mathrm{C}}$ & $0.0 \pm 0.00^{\mathrm{H}}$ & $14 \pm 0.48^{\mathrm{E}}$ & $17 \pm 0.00^{\mathbf{F}}$ & $0.0 \pm 0.00^{\mathrm{H}}$ & $12 \pm 1.47^{\mathbf{G}}$ & $19 \pm 1.44^{\mathbf{D}}$ & $0.0 \pm 0.00^{\mathrm{H}}$ & $11 \pm 0.99^{\mathrm{G}}$ & $11 \pm 0.00^{\mathbf{G}}$ & $0.0 \pm 0.00^{\mathbf{H}}$ \\
\hline Pen. chrysogenum & $25.7 \pm 0.73^{\mathrm{C}}$ & $0.0 \pm 0.00^{\mathbf{H}}$ & $17 \pm 0.48^{\mathbf{F}}$ & $23 \pm 0.00^{\mathrm{C}}$ & $0.0 \pm 0.00^{\mathrm{H}}$ & $14 \pm 1.44^{\mathrm{E}}$ & $26 \pm 0.00^{\mathrm{C}}$ & $0.0 \pm 0.00^{\mathrm{H}}$ & $20 \pm 0.00^{\mathbf{D}}$ & $10 \pm 0.00^{\mathbf{G}}$ & $0.0 \pm 0.00^{\mathrm{H}}$ \\
\hline Sac. cerviceae & $15.3 \pm 0.73^{\mathrm{E}}$ & $0.0 \pm 0.00^{\mathrm{H}}$ & $8 \pm 0.00^{\mathrm{K}}$ & $15 \pm 0.00^{\mathbf{E}}$ & $0.0 \pm 0.00^{\mathrm{H}}$ & $8 \pm 0.00^{\mathrm{K}}$ & $20.7 \pm 1.21^{\mathbf{D}}$ & $0.0 \pm 0.00^{\mathrm{H}}$ & $12 \pm 0.00^{\mathbf{G}}$ & $16 \pm 0.00^{\mathrm{E}}$ & $0.0 \pm 0.00^{\mathrm{H}}$ \\
\hline
\end{tabular}
Data expressed as Mean \pm Standard error; all columns or rows of the different letter are significantly different at $\mathrm{P}<0.05$

Table 4. Influence of Nano-formulation on the inhibitory range of mixed-Lactobacilli total extract

\begin{tabular}{|c|c|c|c|c|c|}
\hline & \multicolumn{3}{|c|}{ Total extract } & \multirow[b]{2}{*}{$\mathrm{C}+$} & \multirow[b]{2}{*}{ C- } \\
\hline & ph & phG & hG & & \\
\hline B. cereus & $56 \pm 1.77^{\mathrm{A}}$ & $50 \pm 0.00^{B}$ & $54 \pm 0.00^{\mathrm{A}}$ & $14 \pm 0.00^{\mathrm{H}}$ & $0.0 \pm 0.00^{\mathrm{M}}$ \\
\hline S. aureus & $39 \pm 0.94$ & $36 \pm 0.15^{\mathrm{C}}$ & $45 \pm 0.75^{\mathbf{D}}$ & $15 \pm 0.70^{\mathrm{H}}$ & $0.0 \pm 0.00^{\mathbf{M}}$ \\
\hline L. monocytogenes & $53 \pm 1.48^{\mathbf{B}}$ & $49 \pm 2.45^{\text {B }}$ & $54 \pm 1.00^{\mathbf{A}}$ & $10 \pm 0.00^{\mathrm{K}}$ & $0.0 \pm 0.00^{\mathbf{M}}$ \\
\hline E. coli & $44 \pm 0.00^{\mathbf{D}}$ & $45 \pm 1.00^{\mathbf{D}}$ & $49 \pm 0.36^{\mathbf{B}}$ & $8 \pm 0.00^{\mathbf{E}}$ & $0.0 \pm 0.00^{\mathbf{M}}$ \\
\hline S. enterica & $52.5 \pm 0.50^{\mathbf{B}}$ & $46.3 \pm 1.50^{\mathrm{D}}$ & $44.4 \pm 2.00^{\mathbf{D}}$ & $10 \pm 0.00^{\mathrm{K}}$ & $0.0 \pm 0.00^{\mathrm{M}}$ \\
\hline Ps. aeruginosa & $36 \pm 0.00^{\mathbf{C}}$ & $37 \pm 1.00^{\mathrm{C}}$ & $48 \pm 0.74^{\mathbf{B}}$ & $0 \pm 0.00^{\mathbf{M}}$ & $0.0 \pm 0.00^{\mathbf{M}}$ \\
\hline Asp. Flavus & $14 \pm 0.35^{\mathrm{H}}$ & $12 \pm 0.35^{\mathrm{G}}$ & $12 \pm 0.65^{\mathrm{G}}$ & $11 \pm 0.77^{\mathrm{G}}$ & $0.0 \pm 0.00^{\mathrm{M}}$ \\
\hline Pen. Chrysogenum & $25 \pm 0.94^{\mathbf{F}}$ & $28 \pm 1.00^{\mathbf{F}}$ & $29 \pm 1.15^{\mathrm{F}}$ & $10 \pm 0.20^{\mathrm{K}}$ & $0.0 \pm 0.00^{\mathrm{M}}$ \\
\hline S. cerviceae & $8 \pm 0.00^{\mathbf{E}}$ & $8 \pm 0.14^{\mathbf{E}}$ & $12 \pm 0.00^{\mathrm{G}}$ & $16 \pm 0.00^{\mathrm{H}}$ & $0.0 \pm 0.00^{\mathrm{M}}$ \\
\hline
\end{tabular}

Data expressed as Mean \pm Standard error; all columns or rows of the different letters are significantly different at $\mathrm{P}<0.05$.

Table 5. CG/MS-identified antimicrobial components of single and mixed-lactobacillus extracts.

\begin{tabular}{l|c|c|l}
\multicolumn{1}{c|}{ Compound } & RT & $\begin{array}{c}\text { Area } \\
\%\end{array}$ & \multicolumn{1}{c}{ Application } \\
\hline 2-Hydroxyisocaproic acid, derivative & 13.23 & 6.42 & Broad spectrum bactericidal, fungicidal (Candida and Aspergillus) \\
\hline 3-Phenyllactic acid, derivative & 18.66 & 2 & Broad and effective antibacterial \& antifungal activity \\
\hline Pyrrolo[1,2-a]pyrazine-1,4-dione, hexahydro-3-(2-methylpropyl)- & 23.77 & 1.11 & Potential antifungal \\
\hline Pyrrolo[1,2-a]pyrazine-1,4-dione, hexahydro-3-(2-methylpropyl)- & 23.66 & 1.12 & Potential antifungal \\
\hline 3-Phenyllactic acid, derivative & 18.65 & 1.39 & Broad and effective antibacterial \& antifungal activity \\
\hline 3-Phenyllactic acid, derivative & 18.63 & 1.79 & Broad and effective antibacterial \& antifungal activity \\
\hline Pyrrolo[1,2-a]pyrazine-1,4-dione, hexahydro-3-(2-methylpropyl)- & 23.69 & 1.63 & Potential antifungal \\
\hline Pyrrolo[1,2-a]pyrazine-1,4-dione, hexahydro-3-(2-methylpropyl)- & 23.70 & 31.82 & Potential antifungal \\
\hline 9-Octadecenoic acid, (2-phenyl-1,3-dioxolan-4-yl)methyl ester, trans- & 28.03 & 0.49 & Antimicrobial \\
\hline Lactic acid & 15.63 & 61.49 & Wide range of applications
\end{tabular}




\subsubsection{Nano-formulation of the mixed-Lactobacilli total extract.}

Total extracts of mixed-Lactobacilli fermentates were loaded on chitosan nanoparticles and undergone an evaluation of inhibitory activity through disc diffusion. Results presented in Table 4 show the influence of applying mixed-Lactobacilli total extracts in Nano form. Other than Asp. flavus and Sac. cerviceae, there is significant progress in the inhibitory potential with all pathogens and food spoiler microbes. The activity against gram-positive bacteria upturns by $15-30 \%$; 17 - 20\%; and $15-39 \%$ in case of ph; phG, and with hG - dependent combinations, respectively. Gram-negative bacteria are excessively inhibited as $9-32 \%, 19-$ $32 \%$, and $7-13 \%$ by ph; phG, and with $\mathrm{hG}$ - dependent combinations, respectively. The highest increment is measured in the case of Pen. Chrysogenum was extra inhibited by $47 \%$, $100 \%$, and $45 \%$ by ph; phG, and with hG - dependent combinations, respectively.

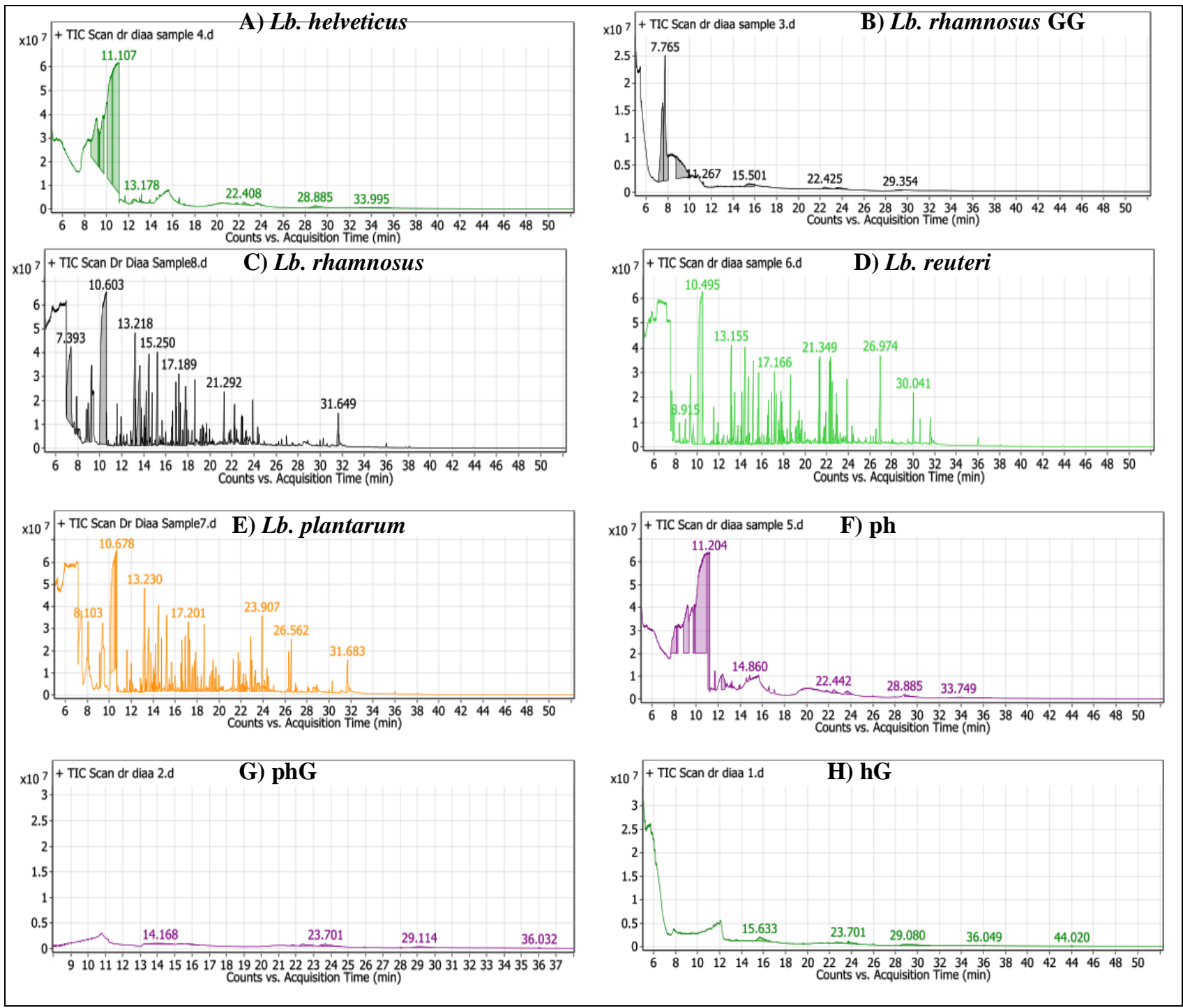

Figure 2. GC/MS chromatograms of different Lactobacilli extracts.

\subsection{Instrumental characterization of bioactive extracts.}

In order to understand the mechanism of antimicrobial action of all obtained active extracts, a set of instrumental investigations were performed to inspect the main components that cause the biological activity of each extract. 


\subsubsection{GC/MS analysis of diethyl ether extract.}

Volatile profile was inspected to explain or understand the effect of each extract against different microbial concerns and resulted in data are supplemented in Table 5 and Figure 2.

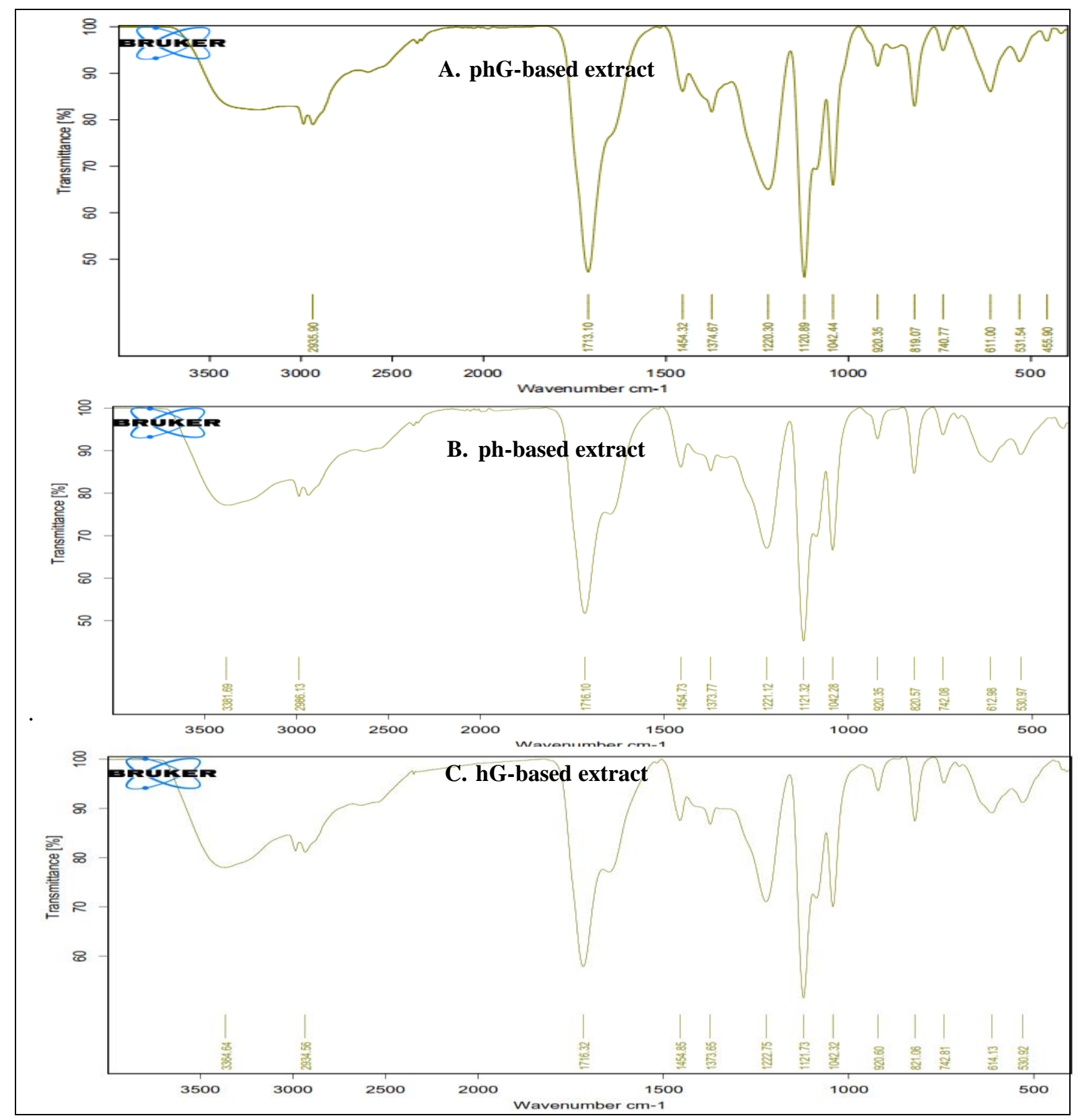

Figure 3. FT-IR spectra of Mixed-Lactobacilli methanol extracts.

Results show that each single or mixed-Lactobacilli DEE extract contains at least a single active compound, with a potential antimicrobial application, besides lactic acid as the main metabolite. In detail, extract of $L b$. plantarum includes 2-Hydroxyisocaproic acid, derivative, and 3-Phenyllactic acid, a derivative that is also produced by both of Lb. rhamnosus and Lb. reuteri. Also, Pyrrolo [1, 2-a] pyrazine-1, 4-dione, hexahydro-3-(2-methylpropyl) - is contained within extracts of $L b$. helveticus, Lb. rhamnosus, ph, and phG-based combinations. A 9-Octadecenoic acid (2-phenyl-1, 3-dioxolan-4-yl) methyl ester trans- was also extracted from phG-based mixture fermentate, while hG-dependent extract only contains Lactic acid. 


\subsubsection{FT-IR spectroscopic analysis of methanol extract.}

Methanol extract of mixed-Lactobacilli fermentations was tested by Fourier transform infrared spectroscopic analysis to predict functional groups of the bioactive components contained within each, and spectra were shown in Figure 3.

Fourier Transform Infra-Red was applied to detect vibration and functional groups contained in methanol extract. The obtained bands at wavenumbers $1650 \mathrm{~cm}^{-1}, 1520 \mathrm{~cm}^{-1}$, and $1310 \mathrm{~cm}^{-1}$, respectively, can be attributed to the presence of amide I, amide II, and amide III of protein while at wavenumber $1454 \mathrm{~cm}^{-1}$ there is asymmetric bending of methyl or methylene group of protein. The bands at the area of $1454 \mathrm{~cm}^{-1}-1221 \mathrm{~cm}^{-1}$ confirmed the existence of the carboxyl group of protein. The presented results of both Table 1 and Figure 3 revealed that the methanol extract seems to bacteriocin-like compound.

\subsubsection{Zeta potential and particle size distribution of prepared Nanoparticles.}

Total extracts of mixed-Lactobacilli fermentation were characterized via zeta potential and particle size distribution facilities. Results are shown in Figure 4. As estimated, the average size and zeta potentials are $260.8 \pm 1.27 \mathrm{~nm}$ and $7.76 \mathrm{mV}$, respectively.

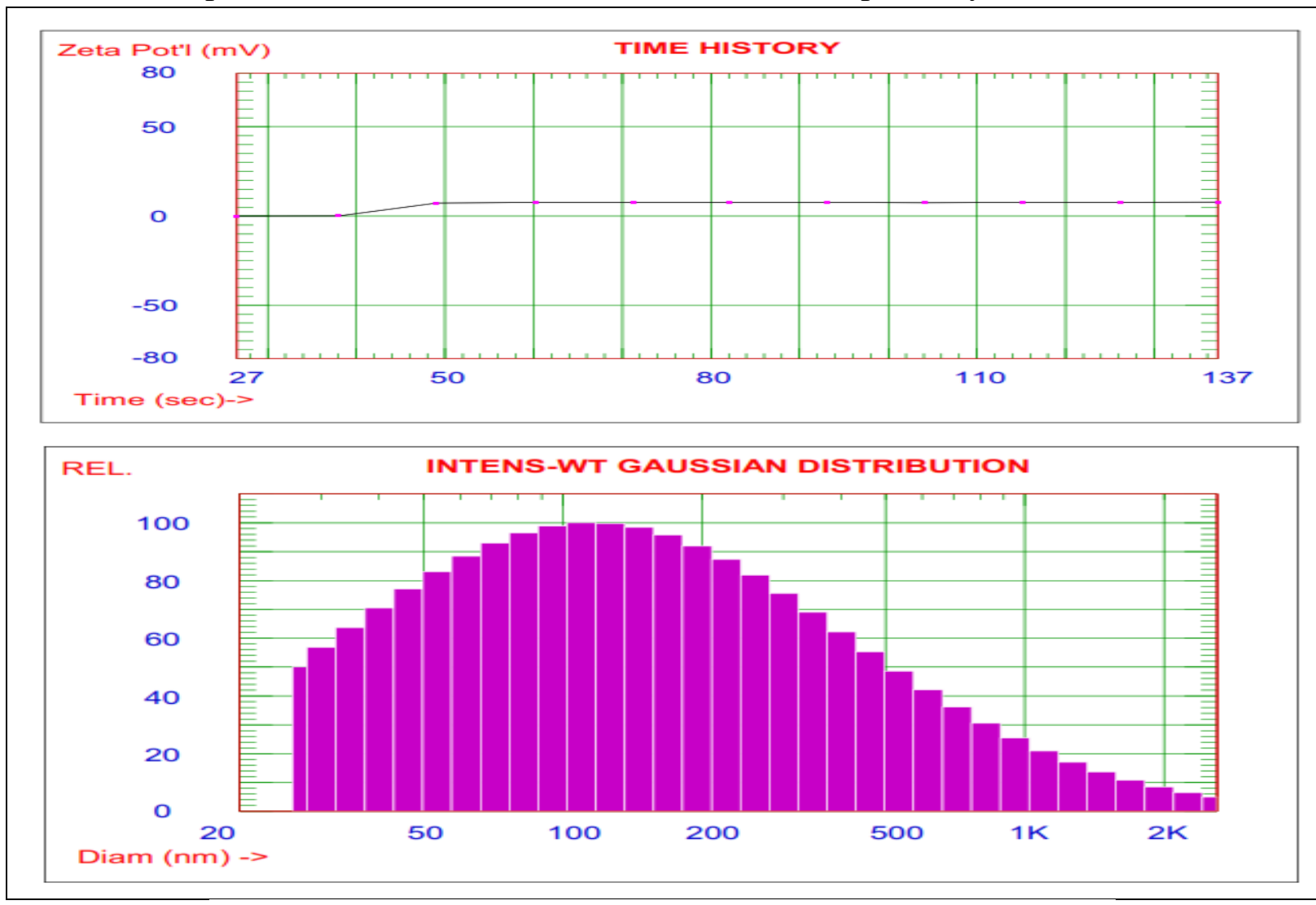

Figure 4. Particle size distribution and zeta potential of phG-based total extract.

\subsection{Discussion.}

The current study is in the way to improve tools of food preservation that based on nature, utilizing five Lactobacillus strains. Different systems extracted fermented Cell-free supernatants, and the obtained extracts were evaluated and characterized.

The results presented in Table 1 summarize the antimicrobial effects of methanol extracted acetone precipitate through disc diffusion. The negative response of fungal populations is that the tested extract (methanol extract of cold acetone precipitate) is strictly 
antibacterial and has no activity against molds and yeasts. From FT-IR spectra that are involved in Figure 3, the obtained bands at wavenumbers $1650 \mathrm{~cm}^{-1}, 1520 \mathrm{~cm}^{-1}$, and $1310 \mathrm{~cm}^{-1}$, respectively, can be attributed to the presence of amide I, amide II, and amide III of protein, while at wavenumber $1454 \mathrm{~cm}^{-1}$ there is asymmetric bending of methyl or methylene group of protein. The bands at the area of $1454 \mathrm{~cm}^{-1}-1221 \mathrm{~cm}^{-1}$ confirmed the existence of the carboxyl group of protein [34]. The presented results of both Table 1 and Figure 3 revealed that the methanol extract seems to bacteriocin-like compound.

From methanol extract to Diethyl ether extract, activity was increased, as well, range of inhibited microbes was extended (Figure 1). As listed in Table (5), this enhancement may be due to the inclusion of at least one antimicrobial compound other than lactic acid. In detail, both Lactobacillus helveticus and Lactobacillus rhamnosus GG with their based-combinations possess the production of Pyrrolo[1,2-a]pyrazine-1,4-dione, hexahydro-3-(2-methylpropyl)which is known as a strong antifungal agent [35]. The observed effect was reported by RochaRamírez et al., [36].

In addition, 3-Phenyllactic acid and derivatives were detected within extracts of Lactobacillus plantarum, Lactobacillus rhamnosus, and Lactobacillus reuteri. This compound was applied as a high potential antibacterial and antifungal compound [37].

The considerable potential of Lactobacillus plantarum as both antibacterial and antifungal can be attributed to the co-production of 2-Hydroxyisocaproic acid and derivatives that are considered a great bactericidal and fungicidal compound [38]. The results in this study come agreed by Arena et al., [39]; they used various strains of Lb. plantarum to control serious food pathogens like Listeria monocytogenes, Salmonella enteritidis, Escherichia coli O157:H7, and Staphylococcus aureus. Also, Lb. plantarum is commonly used to inhibit fungal growth, especially that invade cereals-based products. The results obtained by Arena et al. [40] reported the promising applications of $L b$. plantarum is a probiotic strain to be used in different fields.

Moreover, 2-Hydroxyisocaproic acid and 9-Octadecenoic acid, (2-phenyl-1,3dioxolan-4-yl)methyl ester, trans- contained within ph and phG-based Lactobacillus combinations may be responsible for their increased antimicrobial activity [41]. Nikolova et al., [42] investigated the inhibitory potential of Lactobacillus helveticus strain $50 \mathrm{p} 1$, and results reflected a strong effect against clinical isolates like Pseudomonas aeruginosa and Bacillus sp.. Moreover, there is an agreement with the results of Gomez et al., [43]. They used a biofilm of mixed-lactic acid bacteria to retard the growth of different food-originated human pathogens, such as; Salmonella typhimirium and E. coli O157:H7.

Recent progress in nanotechnology with applicable uses in the food sector, which is rather recent compared with their use in biomedical and pharmaceutical applications. Nanoformulated materials have applications in various food science sectors, including new packaging materials and encapsulated food components [44, 45]. Seeing the application of chitosan in dairy-based foods, Santonicola, et al., [46] used Natamycin-loaded Nano chitosan to pack cheese samples. They reported a significant reduction of mold and yeast content of Nano-treated samples than that of free-state Natamycin. Also, [47, 48] studied the activity of bioactive compound-loaded NPs against some pathogenic bacteria. El- Sayed et al. [49] reported that Nano-encapsulation of bioactive material has strong antimicrobial activity and can extend cheese shelf life during the storage period in all treatments. As recorded in Table (4), the application of mixed-Lactobacilli as Nano-formulated stimulates the increase in their antimicrobial activity by $39 \%, 32 \%$, and $100 \%$ over their crude state in the case of Gram- 
positive, gram-negative, and Penicillium chrysogenum, respectively. The main cause of inhibition enhancement is thought to be the damage of cell membranes after direct contact between Nanoparticles and bacterial membranes [50]. Nanoparticles were reported to show a biocidal effect that covered both gram-positive and gram-negative bacteria [51]. Finally, as seen in the current study, several studies recommended the green production of antimicrobial nanoparticles to protect the environment [52].

The Lactobacillus extracts, particularly the mixed-based, are considered a good tool for controlling or managing pathogenic and food spoilage microorganisms. The inhibitory effect can be magnified if used as Nano-formulated for food preservation and safety maintenance for both food and consumers. This study will help many researchers do further studies to develop the food bio-preservation sector.

\section{Conclusions}

It can be concluded that the current findings recommend the usefulness of the Mixedbased Lactobacillus extract that depends on Lb. helveticus, Lb. plantarum and Lb. rhamnosus $G G$ to inhibit the growth of pathogenic food spoilage bacteria and molds. Additional simulation studies need to validate the effect of this formulation to extend the shelf life of food products. Food preservation by natural methods is an appropriate way to solve economic losses caused by microbial spoilage and reduce foodborne poisoning, including infections and intoxications.

\section{Funding}

This research was funded by National Research Centre, Dokki, Giza, Egypt, under Project Grant No. 12050302.

\section{Acknowledgments}

I would like to thank all respectful colleagues in Marine Toxin Lab. for place availability for solvent extraction.

\section{Conflicts of Interest}

The authors declare no conflict of interest.

\section{References}

1. Buzby, J.C.; Roberts, T.; Lin, C.J.; MacDonald, J.M. Bacterial foodborne disease: medical costs and productivity losses. Food and Consumer Economics Division. Economic Research Service, US Department of Agriculture. Agricultural Economic Report 1996, 741, https://doi.org/10.22004/ag.econ.33991.

2. Zwietering, M.H.; Garre, A.; Wiedmann, M.; Buchanan, R.L. All food processes have a residual risk, some are small, some very small and some are extremely small. Current Opinion in Food Science 2021, 39, 83-92, https://doi.org/10.1016/j.cofs.2020.12.017.

3. Delesa, D.A. Bacteriocin as an advanced technology in food industry. Int. J. Adv. Res. Biol. Sci 2017, 4, 178190, https://doi.org/10.22192/ijarbs.2017.04.12.018.

4. Tayel, A.A.; Bahnasy, A.G.; Mazrou, K.E.; Alasmari, A.; El Rabey, H.A.; Elboghashy, S.A.; Diab, A.M. Biopreservation and quality enhancement of fish surimi using colorant plant extracts. Journal of Food Quality 2021, 2021, 6624565, https://doi.org/10.1155/2021/6624565.

5. Prabhurajeshwar, C.; Chandrakanth, K. Evaluation of antimicrobial properties and their substances against pathogenic bacteria in-vitro by probiotic Lactobacilli strains isolated from commercial yoghurt. Clinical Nutrition Experimental 2019, 23, 97-115, https://doi.org/10.1016/j.yclnex.2018.10.001. 
6. Bergwerff, A.A.; Debast, S.B. Modernization of Control of Pathogenic Micro-Organisms in the Food-Chain Requires a Durable Role for Immunoaffinity-Based Detection Methodology-A Review. Foods 2021, 10, 832, https://doi.org/10.3390/foods10040832.

7. Garnier, L.; Valence, F.; Mounier, J. Diversity and control of spoilage fungi in dairy products: an update. Microorganisms 2017, 5, 42, https://doi.org/10.3390/microorganisms5030042.

8. Odeyemi, O.A.; Alegbeleye, O.O.; Strateva, M.; Stratev, D. Understanding spoilage microbial community and spoilage mechanisms in foods of animal origin. Comprehensive reviews in food science and food safety 2020, 19, 311-331, https://doi.org/10.1111/1541-4337.12526.

9. Shi, C.; Knøchel, S. Sensitivity of Molds From Spoiled Dairy Products Towards Bioprotective Lactic Acid Bacteria Cultures. Frontiers in microbiology 2021, 12, 631730, https://doi.org/10.3389/fmicb.2021.631730.

10. Alp, D.; Bulantekin, Ö.. The microbiological quality of various foods dried by applying different drying methods: a review. European Food Research and Technology 2021, 247, 1-11, https://doi.org/10.1007/s00217-021-03731-z.

11. Giannakourou, M.C.; Tsironi, T.N. Application of Processing and Packaging Hurdles for Fresh-Cut Fruits and Vegetables Preservation. Foods 2021, 10, 830, https://doi.org/10.3390/foods10040830.

12. Todorov, S.D.; Rachman, C.; Fourrier, A.; Dicks, L.M.; Van Reenen, C.A.; Prévost, H.; Dousset, X. Characterization of a bacteriocin produced by Lactobacillus sakei R1333 isolated from smoked salmon. Anaerobe 2011, 17, 23-31, http://doi.org/10.1016/j.anaerobe.2010.01.004.

13. Messaoudi, S.; Manai, M.; Kergourlay, G.; Prévost, H.; Connil, N.; Chobert, J.M.; Dousset, X.. Lactobacillus salivarius: bacteriocin and probiotic activity. Food microbiology 2013, 36, 296-304, https://doi.org/10.1016/j.fm.2013.05.010.

14. Fonseca, H.C.; de Sousa Melo, D.; Ramos, C.L.; Dias, D.R.; Schwan, R.F.; Probiotic properties of lactobacilli and their ability to inhibit the adhesion of enteropathogenic bacteria to caco- 2 and ht-29 cells. Probiotics and antimicrobial proteins 2021, 13, 102-112, https://doi.org/10.1007/s12602-020-09659-2.

15. Yang, K.M.; Kim, J.S.; Kim, H.S.; Kim, Y.Y.; Oh, J.K.; Jung, H.W.; Park, D.S.; Bae, K.H. Lactobacillus reuteri AN417 cell-free culture supernatant as a novel antibacterial agent targeting oral pathogenic bacteria. Scientific reports 2021, 11, 1-16, https://doi.org/10.1038/s41598-020-80921-X.

16. Corr, S.C.; Li, Y.; Riedel, C.U.; O'Toole, P.W.; Hill, C.; Gahan, C.G. Bacteriocin production as a mechanism for the antiinfective activity of Lactobacillus salivarius UCC118. Proceedings of the National Academy of Sciences 2007, 104, 7617-7621, https://doi.org/10.1073/pnas.0700440104.

17. El-Gendy, A.O.; Brede, D.A.; Essam, T.M.; Amin, M.A.; Ahmed, S.H.; Holo, H.; Nes, I.F.; Shamikh, Y.I. Purification and characterization of bacteriocins-like inhibitory substances from food isolated Enterococcus faecalis OS13 with activity against nosocomial enterococci. Scientific reports 2021, 11, 1-12, https://doi.org/10.1038/s41598-021-83357-z.

18. Garneau, S.; Martin, N.I.; Vederas, J.C. Two-peptide bacteriocins produced by lactic acid bacteria. Biochimie 2002, 84, 577-592, https://doi.org/10.1016/s0300-9084(02)01414-1.

19. Halder, D.; Mandal, S. Antibacterial potentiality of commercially available probiotic lactobacilli and curd lactobacilli strains, alone and in combination, against human pathogenic bacteria. Transl. Biomed 2016, 7, 17, https://doi.org/10.21767/2172-0479.100061.

20. Pithva, S.; Ambalam, P.; Dave, J.M.; Vyas, B.R.M. Antimicrobial peptides of probiotic Lactobacillus strains. Science against microbial pathogens: communicating current research and technological advances 2011, 1, 987-91.

21. Cortés-Zavaleta, O.; López-Malo, A.; Hernández-Mendoza, A.; García, H.S. Antifungal activity of lactobacilli and its relationship with 3-phenyllactic acid production. International journal of food microbiology 2014, 173, 30-35, https://doi.org/10.1016/j.ijfoodmicro.2013.12.016.

22. Neal-McKinney, J.M.; Lu, X.; Duong, T.; Larson, C.L.; Call, D.R.; Shah, D.H.; Konkel, M.E. Production of organic acids by probiotic lactobacilli can be used to reduce pathogen load in poultry. PloS one 2012, 7(9), e43928, https://doi.org/10.1371/journal.pone.0043928.

23. Ibrahim, G.A.; Sharaf, O.M.;Al-Gamal, M.S.; Youssef, A.M.; Dabiza, N.M.; El-Ssayad, M.F. Extraction, Evaluation and Structure Elucidation of Bioactive Metabolites of Lactobacillus helveticus CNRZ 32. Biointerface Research in Applied Chemistry 2021, 11, 7677-7688, https://doi.org/10.33263/BRIAC111.76777688.

24. Arena, M.P.; Silvain, A.; Normanno, G.; Grieco, F.; Drider, D.; Spano, G.; Fiocco, D. Use of Lactobacillus plantarum strains as a bio-control strategy against foodborne pathogenic microorganisms. Frontiers in microbiology 2016, 7, 464, https://doi.org/10.3389/fmicb.2016.00464. 
25. Chaudhry, Q.; Scotter, M.; Blackburn, J.; Ross, B.; Boxall, A.; Castle, L.; Aitken, R.; Watkins, R.. Applications and implications of nanotechnologies for the food sector. Food additives and contaminants 2008, 25, 241-258, https://doi.org/10.1080/02652030701744538.

26. Pathakoti K.; Manubolu M.; Hwang H. Nanostructures: Current uses and future applications in food science. Journal of food and drug analysis 2017, 25, 245-253, https://doi.org/10.1016/j.jfda.2017.02.004.

27. Nile, S.H.; Baskar, V.; Selvaraj, D.; Nile, A.; Xiao, J.; Kai, G. Nanotechnologies in food science: applications, recent trends, and future perspectives. Nano-Micro Letters 2020, 12, 1-34, https://doi.org/10.1007/s40820020-0383-9.

28. Lugani, Y.; Sooch, B.S.; Singh, P.; Kumar, S. Nanobiotechnology applications in food sector and future innovations. In Microbial Biotechnology in Food and Health 2021, 2021, 197-225, https://doi.org/10.1016/B978-0-12-819813-1.00008-6.

29. Afify, A.E.M.M.; Romeilah, R.M.; Sultan, S.I.; Hussein, M.M. Antioxidant Activity and Biological Evaluations of Probiotic Bacteria Strains. International Journal of Academic Research 2012, 4, 131-139, https://scholar.cu.edu.eg/sites/default/files/abdelmoneimafify/files/antioxidant_activity_and_biological_eva luations.pdf.

30. Al-Gamal, M.S.; Ibrahim, G.A.; Sharaf, O.M.; Radwan, A.A.; Dabiza, N.M.; Youssef, A.M.; El-ssayad, M.F. The protective potential of selected lactic acid bacteria against the most common contaminants in various types of cheese in Egypt. Heliyon 2019, 5, e01362, https://doi.org/10.1016/j.heliyon.2019.e01362.

31. Matikevičienė, V.; Grigiškis, S.; Lubytè, E.; Dienys, G. Partial Purification and Characterization of Bacteriocin-like Peptide Produced by Staphylococcus xylosus. In Proceedings of the 11th International Scientific and Practical Conference 2017, 3, 213-216, https://doi.org/10.17770/ETR2017VOL3.2586.

32. Andrews, J.M. BSAC standardized disc susceptibility testing method (version 4). Journal of antimicrobial chemotherapy 2005, 56, 60-76, https://doi.org/10.1093/jac/dki124.

33. Williams, L.J.; Abdi, H. Fisher's least significant difference (LSD) test. Encyclopedia of research design 2010, 218, 840-853, http://dx.doi.org/10.4135/9781412961288.n154

34. Odo, I.F.; Ezeanyika, L.U.S.; Ogugua, V.N.; Joshua, P.E.; Okagu, I.U. FTIR and GCMS spectroscopic analysis of methanol and chloroform extracts of Brenania brieyi root bark. AJ Res. Commun 2017, 5, 44-54, www.usa-journals.com.

35. Kannabiran, K. Bioactivity of Pyrrolo [1, 2-a] pyrazine-1, 4-dione, hexahydro-3-(phenylmethyl)-Extracted from Streptomyces sp. VITPK9 Isolated from the Salt Spring Habitat of Manipur, India. Asian Journal of Pharmaceutics 2016, 10, 265-270, http://dx.doi.org/10.22377/ajp.v10i04.865.

36. Rocha-Ramírez, L.M.; Hernández-Chiñas, U.; Moreno-Guerrero, S.S.; Ramírez-Pacheco, A.; Eslava, C.A. Probiotic Properties and Immunomodulatory Activity of Lactobacillus Strains Isolated from Dairy Products. Microorganisms 2021,9, 825, https://doi.org/10.3390/microorganisms9040825.

37. Mu, W.; Yu, S.; Zhu, L.; Zhang, T.; Jiang, B. Recent research on 3-phenyllactic acid, a broad-spectrum antimicrobial compound. Applied Microbiology and Biotechnology 2012, 95, 1155-1163, https://doi.org/10.1007/s00253-012-4269-8.

38. Sakko, M.; Moore, C.; Novak-Frazer, L.; Rautemaa, V.; Sorsa, T.; Hietala, P.; Järvinen, A.; Bowyer, P.; Tjäderhane, L.; Rautemaa, R. 2-hydroxyisocaproic acid is fungicidal for Candida and Aspergillus species. Mycoses 2014, 57, 214-221, https://doi.org/10.1111/myc.12145.

39. Arena, M.P.; Russo, P.; Capozzi, V.; López, P.; Fiocco, D.; Spano, G. Probiotic abilities of riboflavinoverproducing Lactobacillus strains: a novel promising application of probiotics. Applied microbiology and biotechnology 2014, 98, 7569-7581, https://doi.org/10.1007/s00253-014-5837-X.

40. Arena, M.P.; Silvain, A.;Normanno, G.; Grieco, F.; Drider, D.; Spano, G.; Fiocco, D. Use of Lactobacillus plantarum strains as a bio-control strategy against foodborne pathogenic microorganisms. Frontiers in microbiology 2016, 7, 464, https://doi.org/10.3389/fmicb.2016.00464.

41. Adeyemi, M.A.; Ekunseitan, D.A.; Abiola, S.S.; Egbeyale, L.T.; Sogunle, O.M.; Dipeolu, M.A. Phytochemical analysis and GC-MS determination of Lagenaria breviflora R. Fruit. International Journal of Pharmacognosy and Phytochemical Research 2017, 9, 1045-1050, https://doi.org/10.25258/phyto.v9i07.11178.

42. Nikolova, D.; Petrova, M.; Evstatieva, Y.; Danova, S.; Atev, A. Antimicrobial activity of Lactobacillus

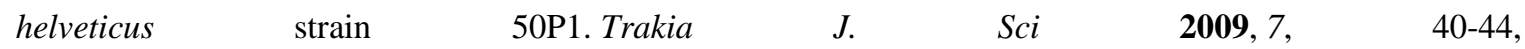
http://citeseerx.ist.psu.edu/viewdoc/download?doi=10.1.1.616.9940\&rep=rep1\&type=pdf.

43. Gómez, N.C.; Ramiro, J.M.; Quecan, B.X.; de Melo Franco, B.D. Use of potential probiotic lactic acid bacteria (LAB) biofilms for the control of Listeria monocytogenes, Salmonella Typhimurium, and 
Escherichia coli O157: H7 biofilms formation. Frontiers in microbiology 2016, 7, 863, https://doi.org/10.3389/fmicb.2016.00863.

44. Gallocchio, F.; Belluco, S.; Ricci, A. Nanotechnology and food: brief overview of the current scenario. Procedia food science 2015, 5, 85-88, https://doi.org/10.1016/j.profoo.2015.09.022.

45. Kiss, É. Nanotechnology in Food Systems: A Review. Acta Alimentaria 2020, 49, 460474, https://doi.org/10.1556/066.2020.49.4.12.

46. Santonicola, S.; Ibarra, V.G.; Sendón, R.; Mercogliano, R.; Rodríguez-Bernaldo de Quirós, A. Antimicrobial films based on chitosan and methylcellulose containing natamycin for active packaging applications. Coatings 2017, 7, 177, https://doi.org/10.3390/coatings7100177.

47. Zohri, M.; Alavidjeh, M.S.; Haririan, I.; Ardestani, M.S.; Ebrahimi, S.E.S.; Sani, H.T.; Sadjadi, S.K. A comparative study between the antibacterial effect of nisin and nisin-loaded chitosan/alginate nanoparticles on the growth of Staphylococcus aureus in raw and pasteurized milk samples. Probiotics and Antimicrobial proteins 2010, 2, 258-266, https://doi.org/10.1007/s12602-010-9047-2.

48. Zohri, M.; Shafiee Alavidjeh, M.; Mirdamadi, S.S.; Behmadi, H.; Hossaini Nasr, S.M.; Eshghi Gonbaki, S.; Shafiee Ardestani, M.; Jabbari Arabzadeh, A. Nisin-Loaded Chitosan/Alginate Nanoparticles: A Hopeful Hybrid Biopreservative. Journal of Food safety 2013, 33, 40-49, https://doi.org/10.1111/jfs.12021.

49. El- Sayed, H. S.; Salama, H. H.; El- Shafei, K.; Effat, B. A.; Tawfik, N. F.; Ibrahim, G.A.; Dabiza, N.; Sharaf, O.M. Comparative Study on Antifungal Activity of Some Nanoencapsulated Natamycin Systems. Middle East Journal of Applied Sciences 2018, 8, 851-855, https://www.curresweb.com/mejas/mejas/2018/851855.pdf.

50. Li, H.; Chen, Q.; Zhao, J.; Urmila, K. Enhancing the antimicrobial activity of natural extraction using the synthetic ultrasmall metal nanoparticles. Scientific reports 2015, 5, 1-13, https://doi.org/10.1038/srep11033.

51. Habtemariam, A.B.; Sibhatu, A.K.; Weldegebrieal, G.K.; Zelekew, O.A.; Tekletsadik, B.T. Bio-mediated synthesis of $\mathrm{ZnO}$ nanostructures from Thymus Schimperi leaves extract and its antibacterial and photocatalytic activities. Letters in Applied NanoBioScience 2020,9, 808-813, https://doi.org/10.33263/LIANBS91.808813.

52. Priya, A.; Saminathan, S.; Balasundaram, J. Green synthesis of NiO nanoparticles using Leucas Aspera and its antibacterial activity. Letters in Applied NanoBioScience 2020, 9, 1033-1036, https://doi.org/10.33263/LIANBS92.10331036. 